woorden, die den jeugdigen leeftijd uitdrukken, zich gaarne in pejoratieve richting beweegt, zoo b.v. ons deerne, meid, boef.

Utrecht.

JOS. SCHRIJNEN.

\title{
LE COUCHER DU SOLEIL EN GRÈCE.
}

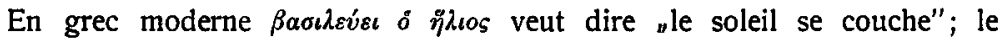

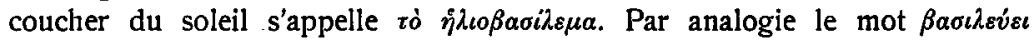
s'emploie dans le même sens en parlant de la lune êt d'autres corps célestes;

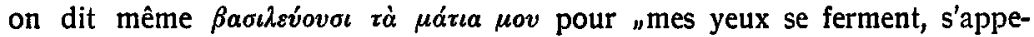
santissent" 1).

Il n'est pas étonnant que cette expression un peu paradoxale ail donné

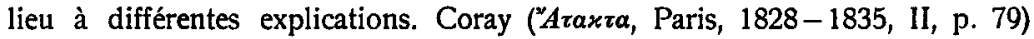

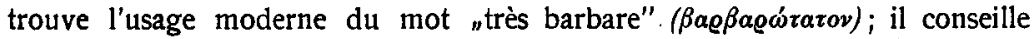

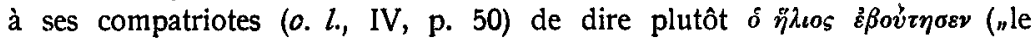
soleil a plongé"). En même temps il donne une explication du prétendu barbarisme: le soir on chante un hymne commençant par les mots $\dot{\delta}$ xv́ @ıos

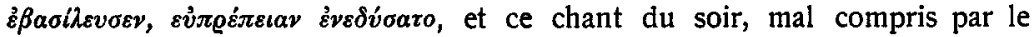
peuple ignorant, aurait provoqué la locution incriminée. Il ne nous paraît pas nécessaire de démontrer combien cette explication de l'illustre savant est forcée et peu vraisemblable.

Grimm, dans sa Deutsche Mythologie (Berlin, 1876, p. 618 note), a indiqué

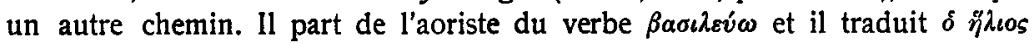

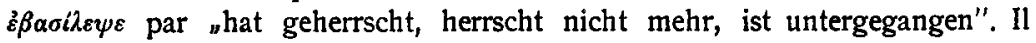
n'en dit pas plus que cela, mais sans doute il a supposé que sur cet aoriste en fonction d'un parfait grec ancien (qu'on compare les deux fonctions du parfait latin) et signifiant "la royauté du soleil a disparu", on ait refait un présent avec la signification "la royauté est en train de disparaître". A mon avis l'hypothèse de Grimm n'est pas soutenable, parce qu'en grec moderne le présent $\beta \alpha \sigma \lambda \varepsilon v i \omega$ n'a jamais un sens qui s'approche de "diminuer, faiblir". Tout au contraire on dit d'une personne qui se porte extrêmement

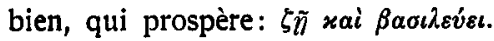

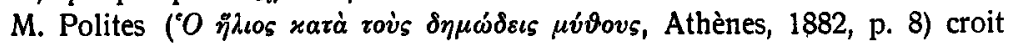

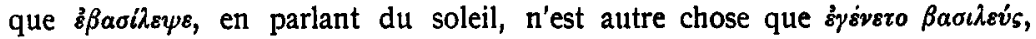
c'est-à-dire: "il a pris possession de son royaume", en allant à son palais situé dans l'extrême Occident, derrière les montagnes, au bout de la terre où se trouvent les champs Élyséens et les îles des bienheureux. - Il est vrai que déjà dans l'antiquité le soleil est appelé un prince, ävoz̧, et que les Hellènes, comme beaucoup d'autres peuples, lui attribuent une demeure splendide; il est vrai encore que d'ordinaire cette demeure est localisée dans

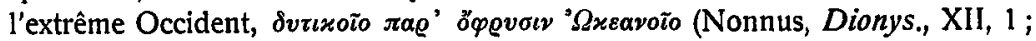
comp. Stésichore, fr. 8, chez Athénée, XI, 469e), mais n'oublions pas que cette localisation n'est pas rigoureuse: on se rappelle que pour Ovide, qui sans doute rapporte une tradition helléniste, la regia solis se trouve en Orient (Metamorph., I, 779; II, 1-18) et que pour Plutarque (Banquet des sept

1) Une telle metaphore n'a rien de surprenant. Qu'on compare en français „il rayonne", "les coups pleuvaient, grêlaient", ou en hollandais "hij daagt op" (il arrive). 
Sages, c. 12 , Moralia p. 155a) le soleil n'a d'autre demeure que son char. Cependant la principale objection qu'on doit faire à $M$. Polites est que le fait de rentrer à la maison, quand même cette maison serait un palais (en

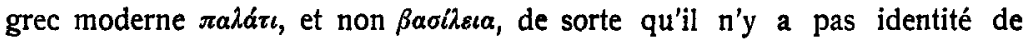
sons), est tout autre chose que commencer à régner; $M$. Polites lui-même

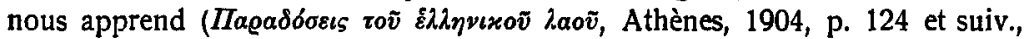
II, p: 806) que le soleil se rend à son palais pour manger et pour s'y reposer pendant quelques heures auprès de sa mère; on pourrait donc plutôt dire que pour un certain temps il dépose la couronne. En outre il est peu probable que le peuple ait cru que le règne du soleil commence au moment où il disparaît à nos yeux. Il $\mathrm{y}$ a donc plusieurs raisons pour rejeter cette explication, malgré tout le respect que nous inspire le meilleur connaisseur du folklore grec.

La locution $\delta \ddot{\eta} \lambda_{\iota o s} \beta a \sigma i \lambda \varepsilon v i \varepsilon$ à un équivalent exact en albanais, ce qui fait supposer à M. Jokl (Mitteilungen des Rumän. Instituts a. d. Univ. Wien, I (1914), p. 298-308) que, dans ce cas, le grec a ţraduit littéralement une manière de s'exprimer albanaise, langue dans laquelle le mot pour "coucher" aurait été mis en rapport, par une parétymologie populaire, avec le mot signifiant "roi" 1 ). La chronologie nous paraît démontrer le mal-fondé de cette supposition. Les Albanais se fixaient en Grèce au XIVe et au XVe siècle; on ne peut pas admettre que déjà au XIVe siècle (voir ci-dessous p. 168) les Grecs aient emprunté aux Albanais cette façon de parler sans se soucier du sens original de $\beta a \sigma c \lambda \varepsilon v \omega$. En grec moderne il n'y a que cinq ou six mots d'origine albanaise, tandis que l'albanais a emprunté plus de huit cents mots à la langue grecque. Il faudra donc considérer la locution albanaise comme une traduction du grec, ainsi que le fait G. Meyer dans son Etymologisches Wörterbuch der albanesischen Sprache (Strasbourg, 1891, p. 328).

Dans l'Annuaire de l'Université d'Athènes (année 1912, p. 2) M. Hatzidakis a fait une observation qui, selon lui, donne la solution de l'énigme. Il nous

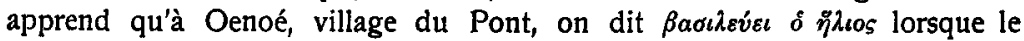
soleil est à son apogée. Rien n'est plus logique: le soleil est alors sur son trône, il règne en roi. Cela doit être l'usage le plus ancien qu'on ait fait de la locution, dit $M$. Hatzidakis, et "partant de cet usage on l'a transporté

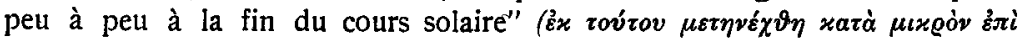

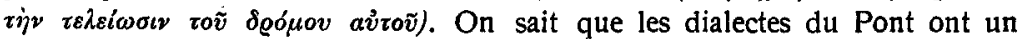
caractère très archaïque ${ }^{2}$ ); sur plusieurs points ils présentent un état linguistique qu'a dépassé depuis bien des siècles la langue commune. Je crois donc avec $M$. Hatzidakis que l'usage signalé par lui est le plus ancien, mais je n'y vois qu'une étape, importante il est vrai, vers l'explication. On se demande pourquoi ou comment ce transfert du sens dont parle $M$. Hatzidakis s'est opéré. Il donne comme un cas analogue le fait qu'en crétois le mot $\partial\left\langle\varepsilon^{\prime} \varsigma\right.$ (de ö $\psi \varepsilon$ ) a pris d'abord la signification de "veille" (qu'il a encore à

1) Cette parétymologie me paraît peu probable, mais je n'ai aucune compétence en matière d'albanais.

2) J'ai cherché en vain dans les autres dialectes de la périphérie des exemples de cet emploi de

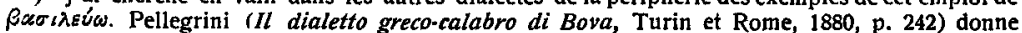
vasileggui et vasilemma dans le sens de tramontare, tramonto. Il déclare ne pas compendre l'origine de cette expression. 
Corfou) et puis celle de "hier". On pourrait de la même façon citer le français "veille", devenu de "nuit qui précède une fête religieuse", "jour qui précède un jour déterminé" ${ }^{1}$ ). Dans les deux cas on constate l'extension d'un concept qui s'y prête par sa nature même: ŏ $\psi \varepsilon$, "tard", est une notion de temps peu précise et l'on peut commencer une veillée de tres bonne heure. Pour $\beta a \sigma i \lambda \varepsilon v ́ \omega$ le cas nous paraît différent, surtout parce que ce verbe a peu perdu de sa signification originale. $M$. Hatzidakis, prévoyant cette objection, suggère que ce serait la confusion supposée par Coray qui aurait amené la transition de sens. Mais nous avons déjà dit que cette confusion nous semble tout à fait invraisemblable. Pour serrer la question de plus près, il sera bon de nous rappeler l'histoire, d'ailleurs très simple, du mot $\beta a \sigma i \lambda \varepsilon v$ w.

En grec classique $\beta a \sigma i \lambda \varepsilon v ́ \varepsilon \imath$ veut dire "être roi, régner" 2). Dans ce sens le verbe est resté en usage jusqu'à nos jours, mais surtout dans la langue officielle, qui est basée sur la tradition écrite. Jannaris (An historical greek

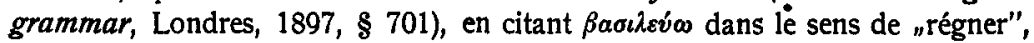
pourvoit le verbe d'un signe dénotant les mots anciens "encore vivants, mais seulement en partie ou dans un sens modifié" ("still surviving but only partially or in a modification"). Pour lui le sens vraiment populaire du mot est "coucher" ("to go down, to set", o. $l$., $\$ 701 b$ ). Cependant nous avons déjà vu que dans la langue parlée $\beta a \sigma \iota \lambda \varepsilon v ́ \omega$ signifie aussi „vivre en roi, prospérer" (voir ci-dessus, p. 165). Ce sens est un développement de celui de "régner". Le premier exemple que j'en connaisse se trouve dans Théocrite. Un pauvre pêcheur voit en songe un poisson d'or qu'il a capturé, et il jure de ne plus s'aventurer sur la mer mais de rester sur terre et "de

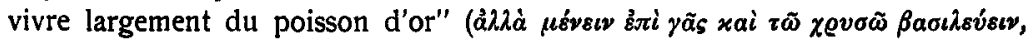
Theocr. XXI, 60) ${ }^{3}$ ). Les exemples de cet usage de $\beta a \sigma i d \varepsilon v i \varepsilon v v$ sont fréquents dans la traduction des Septante (exemples: Proverbes IX, 6, Jérémie XXII, 15) et dans le Nouveau Testament. Toutefois dans ce texte les passages en question contiennent en même temps une allusion au règne auquel participeront les croyants dans le royaume de Dieu. Ainsi dans les paroles de

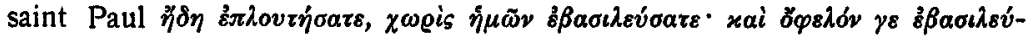

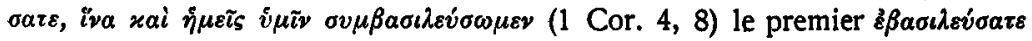
veut dire "vivre en roi", tandis que le second se rapporte au règne des élus.

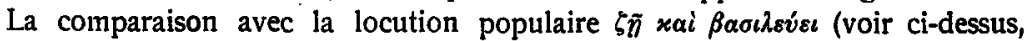
p. 165) montre que dans l'Apocalypse (XX, 4; comp. XX, 6, XXII, 5) les

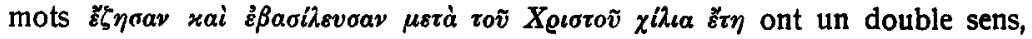

1) M. Kretschmer (Glotta, V, p. 289), en citant l'explication de M. Hatzidakis, fait remarquer que chez les habitants de Hessen, „der Ausdruck Mittag aut den Nachmittag ausgedehnt wird, so daß z. B. 5 Uhr Nachmittags von den Hessen als Mittags bezeichnet wird, also schon eine Stunde in der im Winter die Sonne untergeht"'. On observe le même contresens dans le français "un second déjeuner"; en hollandais on parle couramment d'un "middagmaal" qui commence à six ou sept heures du soir: on ne pense pas a l'étymologie de ces mots.

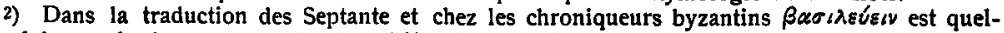
quefois employé comme verbe transitif, "faire quelqu'un roi" (I Livre des Rois VIII, 22, XII, 1 etc.; voir Psaltes, Grammatik der byzantin. Chroniken, Göttingen, 1913, " p. 38 et comp.

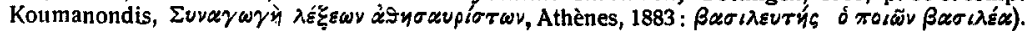

3) Nous n'avons pas à discuter ici l'authenticité suspectée de cette idylle, problème sur lequel on peut consulter le beau livre de $\mathrm{M} \mathrm{Ph}$. E. Legrand, Étude sur Théocrite, Paris'

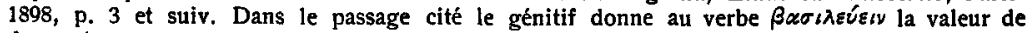
$\dot{x} \pi 0 \lambda x u_{\varepsilon i v}$ 
ce qui sans doute aurait été remarqué par les commentateurs s'ils avaient

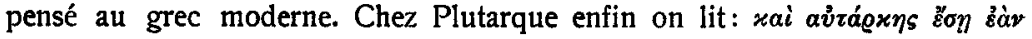

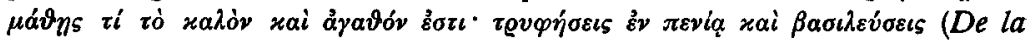
vertu et du vice, c. 4, Moralia, p. 101,d).

Voyons maintenant ce que les textes nous apprennent sur l'emploi de

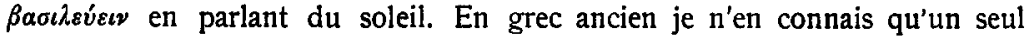
exemple:- dans le Banquet des sept Sages, c. 12 (Plut., Moralia, p. 155a) Anacharsis prétend que le soleil, ayant pour toute demeure son char, est le

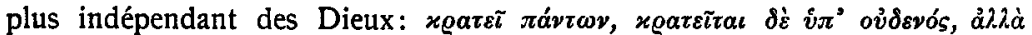

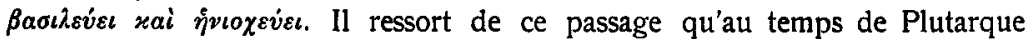

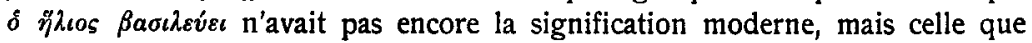
M. Hatzidakis a signalée comme survivant dans un dialecte du Pont. Dans les textes du moyen âge au contraire $\beta a \sigma i \lambda \varepsilon v \varepsilon \iota$ se dit seulement du soleil qui se couche. Voici, dans l'ordre chronologique, les passages qui me sont connus:

Histoire des Quadrupèdes (Wagner, Carmina graeca medii aevi, Leipzig,

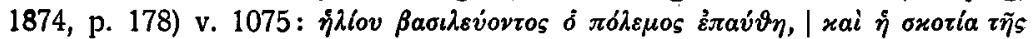

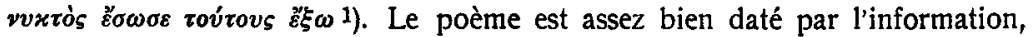
donnée aux vers $11-13$, que la querelle des animaux eut lieu le $15 \mathrm{Sep}-$ tembre 1365. Voir Psichari, Essais de gramm. hist. néo-grecque, II, Paris, 1889, p. 244 et Krumbacher, Gesch. der byzant. Litt., p. 879).

L'Achilléide byzantine (ed. Hesseling, Amsterdam, 1919, p. 110) v. 744

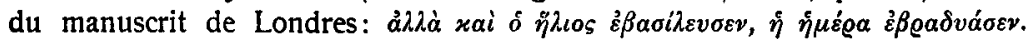
Le manuscrit est du XVe siècle; le manuscrit de Naples (daté du 5 Mai 1520) offre au passage correspondant (v. 1050) $\varepsilon^{\varepsilon} \delta v v \varepsilon v$, celui d'Oxford (écrit au

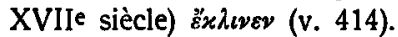

Erotopaegnia (ed. Hesseling et Pernot, Biblioth. grecque vulg., X, p. 20)

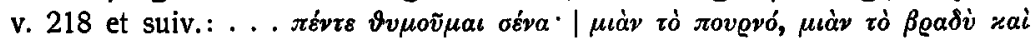

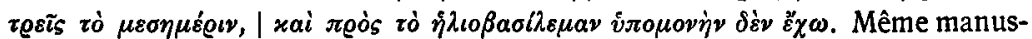
crit que celui de l'Achilleïde (XVe siècle).

La Bataille de Varna (ed. Legrand, Collection de mon. N. S., no. 5):

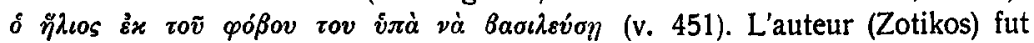
témoin de la bataille (1444).

La Peste de Rhodes (ed. Legrand, Biblioth. grecque vulg. I, p. 216) v. 398

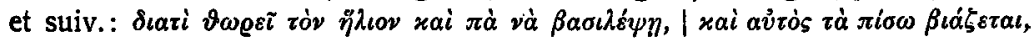

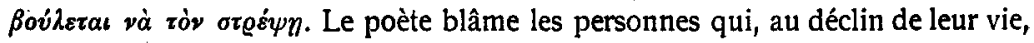
convolent en secondes noces. Le poème contient le récit d'un témoin oculaire du désastre qui frappa Rhodes en 1498; le manuscrit est du XVIe siècle.

Avec ce dernier texte nous nous rapprochons des temps modernes où

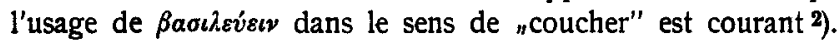

1) Leçon du Ms. de Vienne. Dans un manuscrit de Paris (fonds grec 2911) les mots suivants

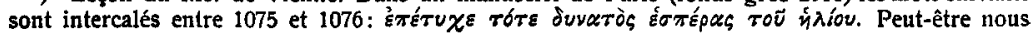

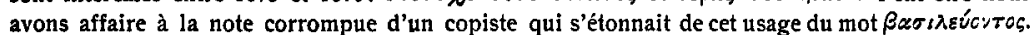

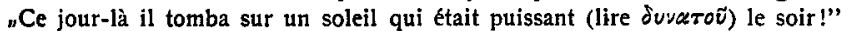

2) Du Cange, dans son Olossaire (Lyon, 1688, p. 180), traduit $\beta \alpha \sigma$ ) $\lambda \varepsilon u ́ \varepsilon u$ par "oriri", et

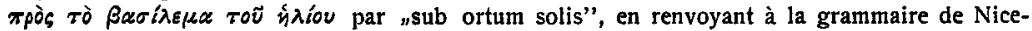
phorus Romanus, qui à cette époque n'existait qu'en manuscrit. Depuis, cette grammaire a été publiée par M. J. Boyens (Biblioth. de la faculté de philosophie et lettres de Liège, fasc. 18, 1908); on y lit, à la page 16, les mots cités par Du Cange, mais traduits par circa yesperam. Du Cange s'est donc trompé. 


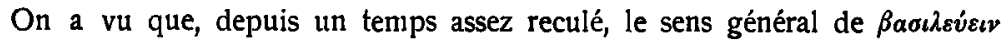
est devenu dans la langue parlée "vivre en roi, prospérer". Ce léger changement de signification va nous donner, je l'espère, la solution du problème.

On sait que le soleil en se couchant paraît beaucoup plus grand que lorsqu'il est plus éloigné de la terre. Ce phenomène, dont je laisse l'explication aux astronomes, s'observe aussi chez nous, mais dans l'air pur de la Grèce il frappe l'esprit d'une toute autre façon. Là il y a quelque chose de stupéfiant dans la splendeur solaire des derniers moments de la journée; l'œil, qui pendant le jour s'abritait contre les rayons' destructeurs, voit alors un astre qui a augmenté en grandeur et dont la disparition ne suggère aucune idée de dépérissement. Le Dieu de la lumière, tout resplendissant de pourpre et d'or, achève son parcours dans la plénitude de son bonheur; en quittant la terre il montre sa majesté, sa $\beta$ acileia. S'il ne règne plus, il est roi plus que jamais. Que ceux qui ne peuvent pas recourir à des souvenirs personnels de ce spectacle inoubliable, se rappellent les célèbres vers qu'il a inspirés à Byron lorsqu'il visita l'Acropole au printemps de 1811 . Je n'en citerai que les quatre premiers:

Slow sinks, more lovely ere his race be run,

Along Morea's hills the setting sun;

Not, as in northern climes, obscurely bright,

But one unclouded blaze of living light.

(Byron, The Corsair, Canto III, v. 1 et suiv.) ${ }^{1}$ ).

Il est vrai que l'agrandissement apparent du soleil se produit aussi bien à son lever qu'à son coucher, mais dans le premier cas il est moins frappant, parce que c'est en quelque sorte un nouveau soleil qu'on voit, dont la grandeur se prête donc moins à la comparaison. Et n'oublions pas que, même parmi des pêcheurs et des paysans, le soleil qui se lève n'est pas au même degré un spectacle de tous les jours que le soleil qui se couche.

Si mon explication de la locution grecque est juste, on peut tirer, ce me semble, une "morale" de la petite histoire que je viens d'esquisser. La voici : il est difficile, sinon impossible, de définir par une formule précise un terme, assez simple en apparence, pour en fixer l'usage pour un temps donné. Des nuances à peine perceptibles se glissent dans l'usage et provoquent quelquefois des changements de sens qui rendent le mot méconnaissable ${ }^{2}$ ).

\section{Leiden.}

D. C. Hesseling.

1) Mon collègue, Mile C. Serrurier, me fait observer que, dans La Henriade (ch. VI, v. 367 et suiv.), Voltaire a chanté le coucher du soleil en termes analogues: ... . ainsi que l'astre auteur de la lumière, | Après avoir rempli sa brûlante carrière, | Au bord de l'horizon brille d'un feu plus doux, | Et, plus grand à nos yeux, paraît fuir loin de nous..." La plus grande intensité du soleil grec se retrouve dans les vers du poète anglais.

2) Voici deux autres exemples du même phénomène: xzvóva, vider - verser d'un vase dans un autre - remplir (dial. crétois); $\sigma \omega ́$ vo, sauver, garder - atteindre - achever, épuiser. 